Check for updates

Cite this: RSC Adv., 2019, 9, 31819

\title{
Hydrophobic resin treatment of hydrothermal autohydrolysate for prebiotic applications
}

\author{
Derek B. Corbett, Changyoung Hong, Richard Venditti, Hasan Jameel \\ and Sunkyu Park (DD *
}

The production of a high-value xylooligosaccharide (XOS) prebiotic product from lignocellulosic autohydrolysate requires processing for the removal of non-carbohydrate components such as lignin and furfural. In this research, the nature of XOS dissolved in autohydrolysate is evaluated including the XOS degree of polymerization (DP) distribution and potential covalent association between XOS and lignin (LCC). The impact of these factors on the yield of XOS during treatment of Miscanthus autohydrolysate with hydrophobic resin is assessed. Over 30\% of the XOS in autohydrolysate was found to be likely associated with lignin ("tied" XOS), all of which was removed during hydrophobic resin treatment along with over $90 \%$ of the dissolved lignin. However, loss of dissolved XOS during resin treatment was found to not be due solely to XOS association with lignin. Over $50 \%$ of the "free," nonlignin-associated XOS was also removed by resin treatment. Interaction between "free" XOS and the hydrophobic resin was found to be highly dependent on DP with higher DP XOS being removed far more readily than low DP XOS. Over $80 \%$ of dissolved "free" XOS with a DP of six and above (X6+) was removed from autohydrolysate during treatment while only $17 \%$ of xylose (X1) was removed. Efforts to understand the interaction between the hydrophobic resin and XOS and to improve the recovery of XOS during hydrophobic resin treatment are presented.

Received 2nd August 2019

Accepted 29th September 2019

DOI: 10.1039/c9ra06018a

rsc.li/rsc-advances extensively for the pretreatment of lignocellulosic biomass. ${ }^{8-14}$ The autohydrolysis process employs water and heat to cleave acetyl groups and other organic acids present, primarily on hemicelluloses. The process is simple, relatively low cost, and effective at improving the pretreated solids for further processing. Much research has been devoted to optimizing the conditions of autohydrolysis for xylan dissolution and XOS production. Previous reports have shown the dissolution of up to $75 \%$ of xylan present in sugar maple hardwood during autohydrolysis $\left(175^{\circ} \mathrm{C}\right.$ for 2 hours) ${ }^{15} \mathrm{XOS}$ yield reached as high as $51 \%$ (but at less severe conditions: $152{ }^{\circ} \mathrm{C}$ for 4.5 hours). ${ }^{15}$ $77 \%$ of the original xylan was solubilized during autohydrolysis of apricot pit shells $\left(160{ }^{\circ} \mathrm{C}\right.$ for 2 hours $) \cdot{ }^{16} \mathrm{~A}$ maximum xylan solubilization of $\sim 70 \%$ was reported during autohydrolysis of Miscanthus $x$ giganteus. ${ }^{17}$ XOS have been reported to account for between 32 and 52 percent of the dry mass of dissolved solids in autohydrolysate (AH) from Miscanthus $x$ giganteus depending on experimental methods used. ${ }^{18,19}$

The autohydrolysis liquor produced from hardwoods and grasses contains a heterogeneous solution of hemicellulosederived monomers and oligosaccharides, lignin, hemicellulose degradation products (furfural and hydroxymethylfurfural), and organic acids (acetic acid, formic acid, glucuronic acid). ${ }^{20}$ While unmodified lignin is not usually soluble in water, it is believed that the close association of this lignin with carbohydrates increases its hydrophilicity and, 
therefore, solubility in water. ${ }^{21-23}$ These dissolved compounds have significant potential for conversion into high-value products. ${ }^{21,24}$ However, the complex, heterogeneous nature of the dissolved materials in pretreatment liquors complicates the production of value-added products. ${ }^{25} \mathrm{~A}$ better understanding of the nature of the relationship between lignin and carbohydrates is important for the further development of economically viable technologies for valorization of lignin/carbohydrates derived from autohydrolysis liquor.

Efforts to purify XOS from autohydrolysate liquor have been undertaken previously. ${ }^{24,26,27}$ Methods that have been pursued for refining autohydrolysate to produce XOS include solvent extraction, solvent precipitation, ion-exchange, flocculation, adsorption, ultra-filtration, and freeze drying. ${ }^{26}$ Vegas and coworkers reported on a processing scheme for the purification of XOS from rice husk autohydrolysate. ${ }^{26}$ Nanofiltration was successful at removing some non-saccharide components from the liquor while also concentrating the solution. A subsequent double ion-exchange process (tested with and without prior solvent extraction) was shown to substantially reduce the noncarbohydrate non-volatile compound content in the liquor. A yield of $\sim 10 \%$ (based on total oven-dried rice husk solids) was reported for a product containing less than $6 \%$ noncarbohydrate impurities. The overall yield of XOS was $\sim 69 \%$ (as a percent of the XOS present in the starting liquor).

Hydrophobic resin has been identified as a low cost method for removal of aromatic non-carbohydrate components from autohydrolysis pretreatment byproduct streams. ${ }^{21}$ Hydrophobic resins are used commercially for many applications where selective adsorption of aromatic or hydrophobic chemicals is desired. Resins can be regenerated relatively easily and require comparatively simple equipment compared with membrane and extraction processing methods. Current industrial applications for hydrophobic resins include high-value applications such as the recovery of products from fermentation broth and lower-value applications such as absorption of phenols from waste water streams. ${ }^{28}$

Narron et al. reported on the use of hydrophobic resin for recovery of dissolved lignin from maple and sugarcane bagasse autohydrolysates. Lignin recovery was over $90 \%$, however, it was reported that the recovered solid adsorbate was highly contaminated with carbohydrates $(48.3 \%$ by weight $) .{ }^{21}$ In addition, while monomeric xylose was not found to be removed by hydrophobic resin, around a third of the oligomeric xylose (determined by acid hydrolysis and subtraction) present in the raw autohydrolysate was removed. ${ }^{21}$ The authors hypothesized that the adsorption of oligomeric xylose and the associated sugar contamination in the adsorbate solid might be due to covalent association between lignin and XOS (LCC). However, that hypothesis was never fully explored in their work.

Huang et al. also investigated the processing of autohydrolysates (sweetgum) utilizing hydrophobic resin for lignin removal. They reported $\sim 7 \%$ loss of XOS (after a substantial water wash) and minor contamination of the recovered lignin with XOS $(\sim 6 \%)$. In their report, as well as in the work by Vegas et al., covalent association between lignin and oligosaccharides by LCC linkages and the impact of XOS degree of polymerization (DP) on autohydrolysate processing was not considered. In fact, in general, these issues have been neglected in related literature up to this point.

The present work investigates the process of adsorption with hydrophobic resin in more depth to determine the potential role of LCC and DP on XOS adsorption. A novel strategy is employed to quantify the "free" and "tied" fractions of xylose and XOS in autohydrolysate. Additionally, the recovery of oligosaccharides during the treatment process is examined for individual DPs, revealing challenges and opportunities that must be considered when designing a process for commercial implementation. Finally, options to improve adsorption selectivity are explored.

\section{Materials and methods}

\subsection{Biomass pretreatment and autohydrolysate liquor wash}

Miscanthus biomass (Pennsylvania, US) was milled down to $\sim 0.5$ inch size. Biomass was pretreated using an AdvanceBio continuous screw reactor ( $2 \mathrm{~kg}$ per hour capacity) at Pennsylvania State University. The pretreatment conditions were as follows: liquorto-wood ratio of $4: 1$, reaction temperature of $190{ }^{\circ} \mathrm{C}, 15 \mathrm{~min}$ residence time. The pretreated biomass was stored in a cold room at $4{ }^{\circ} \mathrm{C}$ until use. To effectively extract dissolved materials, the pretreated biomass was soaked in de-ionized water at room temperature. Vacuum filtration was then performed to remove the pretreated solids producing the autohydrolysate liquor used for all subsequent work in this study.

\subsection{Hydrophobic resins}

Three AMBERLITE ${ }^{\mathrm{TM}} \mathrm{XAD}^{\mathrm{TM}}$ resins (DOW Chemical) were purchased from Sigma Aldrich and used in this study: AMBERLITE $^{\mathrm{TM}} \mathrm{XAD}^{\mathrm{TM}} 4, \quad$ AMBERLITE ${ }^{\mathrm{TM}} \mathrm{XAD}^{\mathrm{TM}} 16 \mathrm{~N}$, and AMBERLITE $^{\text {TM }}$ XAD $^{\text {TM}} 1180$ N. Most analyses were performed using XAD16N. XAD4 and XAD1180N were included to test the impact of XAD physical properties such as specific surface area, porosity, particle size, and average pore diameter on $\mathrm{AH}$ treatment. AMBERLITE XAD is a non-ionic macroreticular crosslinked aromatic polymer resin utilized for a multitude of applications where adsorption of hydrophobic molecules is desired. Hydrophobic resin has been proposed as an option for selective removal of lignin from pretreatment liquor streams. $^{21,29}$

$\mathrm{XAD}$ resins are shipped coated with preservative salts. To remove these salts, an extensive washing procedure was conducted: (1) DI water wash until $\mathrm{pH}$ of wash water is neutral ( $\sim 2000 \mathrm{~mL}$ ), (2) extensive methanol wash to remove an adsorbed impurity ( $\sim 500 \mathrm{ml})$, and (3) water wash to remove methanol $(\sim 500 \mathrm{ml})$. Prepared resins were stored in a refrigerator in a sealed bag. Moisture content of the resin was determined prior to use (typically $\sim 70 \%$ moisture).

\subsection{Adsorption procedure}

Adsorption experiments with hydrophobic resin were performed as previously reported. ${ }^{21}$ Hydrophobic resin loading was 10 OD grams resin/100 $\mathrm{ml}$ of autohydrolysate liquor. Contact time was 30 
minutes in a constant stirring apparatus ( 75 RPM). Multiple $100 \mathrm{~mL}$ water washes were conducted after adsorption to remove any weakly bound compounds. An extensive methanol wash was then performed to recover adsorbed compounds from the hydrophobic resin. The methanol was evaporated using a rotary evaporator and the remaining solids were re-dissolved in water for analysis (or in dimethyl sulfoxide $\mathrm{d}_{6}$ for NMR analysis). When $\mathrm{pH}$ adjustment was needed, $2 \mathrm{~N}$ sodium hydroxide was utilized. Dilution of the sample by water already present in the resin or by $\mathrm{pH}$ adjustment was considered.

\subsection{Compositional analysis}

"Raw" autohydrolysate liquor, hydrophobic resin "cleaned" autohydrolysate liquor, and adsorbate samples were exposed to acid hydrolysis following the NREL procedure for determination of sugars in liquid fraction process samples. ${ }^{30}$ Briefly, the appropriate volume of $72 \%$ sulfuric acid was added to reach $4 \%$ sulfuric acid concentration. Acidified liquor was autoclaved at $121{ }^{\circ} \mathrm{C}$ for 60 minutes. After hydrolysis the hydrolyzed sample was neutralized $\left(\mathrm{CaCO}_{3}\right)$, filtered $(0.2 \mu \mathrm{m}$ nylon filters), and analyzed by HPLC. The HPLC set up was as follows: Shodex SP0810 column ( $8 \AA 200 \mathrm{~mm}$, Showa Denko, Japan) maintained at $80{ }^{\circ} \mathrm{C}$. Milli-Q water mobile phase, flowing at $0.5 \mathrm{~mL} \mathrm{~min}{ }^{-1}$ (60 min per sample). A refractive index detector was used for detection of sugars in eluent. Calibration was performed with commercial standards of glucose, xylose, galactose, mannose, and arabinose. Due to co-elution of mannose and arabinose both are reported together.

Xylose and xylooligosaccharide (XOS) composition was determined in two ways. For compositional analysis of the xylose and XOS content in autohydrolysate, HPLC was utilized. Oligosaccharide content was determined as the difference in monomer concentration before and after acid hydrolysis. For analysis of xylose and XOS adsorption onto hydrophobic resin, and for determination of "free" and "tied" xylose and XOS concentrations in autohydrolysate (terms explained in discussion section), only ion-chromatography (IC) was utilized (even for analysis of xylose monomer, which can be determined by HPLC). This is important, because the two methods for determining xylose content (HPLC and IC) result in slightly different results. The IC system was set up as follows: CarboPac PA200 column held at $30^{\circ} \mathrm{C}$. A 60 minute gradient elution starting with $100 \mathrm{mM} \mathrm{NaOH} / 180 \mathrm{mM}$ sodium acetate solution and ending with $100 \mathrm{mM} \mathrm{NaOH} / 550 \mathrm{mM}$ sodium acetate eluent solution. Eluent solutions were produced with ultra-pure DI water. Detection was achieved with a pulsed amperometric detector (PAD). Since X1-X5 model xylooligosaccharides were used as standards for IC, quantification of XOS with degrees of polymerization (DPs) higher than 5 was estimated. DP $>6$ are presented as X6+. To quantify these higher DP oligosaccharides we used a method similar to that of Yang and Wyman ${ }^{31}$ wherein the calibration factor was used from X5 to quantify X6 and above. Carbohydrate standards, xylose, xylobiose, xylotriose, xylotetraose, xylopentaose, cellobiose, and dextran $\left(M_{\mathrm{r}} \sim 1500\right)$ were purchased from Megazyme. All compositional analyses were completed in at least duplicate.

\subsection{D-HSQC NMR analysis}

2D-HSQC NMR results were acquired as previously described. ${ }^{21}$ Briefly: $100 \mathrm{mg}$ of dry hydrophobic resin adsorbate solid was completely dissolved in $0.5 \mathrm{ml}$ dimethyl sulfoxide- $\mathrm{d}_{6}$ solvent (20\% solution). 2D-HSQC NMR spectra were acquired using a $500 \mathrm{MHz}$ NMR spectrometer equipped with $5 \mathrm{~mm}$ BBI probe (Avance, Bruker, USA) at $25{ }^{\circ} \mathrm{C}$ (140 scans).

\section{Results and discussion}

\subsection{Autohydrolysate characterization}

Pretreatment followed by a room temperature wash resulted in the solubilization of $\sim 22 \%$ of the raw Miscanthus biomass. Over $50 \%$ of the xylan in Miscanthus was recovered in the liquor after washing as either xylose or XOS. Hot water pretreatments such as autohydrolysis are known to extract hemicelluloses from lignocellulosic biomass, while leaving the cellulosic portion relatively untouched. ${ }^{14}$ The removal of xylose is believed to have been relatively low compared to other reports due to the low temperature of the washing process utilized (room temperature). It is likely that higher DP XOS were not effectively solubilized. Around $19 \%$ of the xylose present as xylan in the starting biomass was unaccounted for in the pretreatment solids and liquor. This is believed to be due at least partially to the production of furfural and other degradation products from xylan during autohydrolysis (Table 1).

The Miscanthus autohydrolysate was analyzed for monomeric and oligomeric sugars before and after acid hydrolysis (Table 2). Xylose and xylan were the main monomeric and oligomeric components present in the autohydrolysate as expected based on previous reports on the composition of Miscanthus. ${ }^{18}$ The small presence of glucose monomers is likely derived from glucuronoxylan hemicelluloses. Oligosaccharides accounted for almost half of the total dissolved solids $(\sim 46 \%)$ with the dominant oligosaccharide being xylooligossaccharide (XOS; $\sim 37 \%$ ).

Non-carbohydrate components make up $\sim 25 \%$ of the composition of the autohydrolysate solids. The most notable non-carbohydrate components are acetic acid and dissolved lignin (15.3\% and $7.8 \%$, respectively). The acetic acid is derived from acetyl groups on hemicelluloses, which is one of the major reasons for the $\mathrm{pH}$ reduction during autohydrolysis. The $\mathrm{pH}$ of the autohydrolysate liquor was 3.2 , which is comparable to autohydrolysis liquors from woody hardwoods. ${ }^{22}$ The lignin content in the autohydrolysate liquor (determined by UV analysis at $205 \mathrm{~nm}$ ) was $\sim 7.8 \%\left(1.94 \mathrm{mg} \mathrm{ml}^{-1}\right)$. Approximately $5 \%$ of the total non-volatile solids remained uncharacterized.

\subsection{Xylooligosaccharide present in Miscanthus autohydrolysate}

A combination of acid hydrolysis and chromatography was employed to analyze xylose and XOS in Miscanthus autohydrolysate. We define the term "free" xylose as xylose detected by IC prior to acid hydrolysis. Similarly, we define "tied" xylose as the additional xylose detected by IC after complete acid hydrolysis ("tied" xylose was calculated by subtracting "free" xylose 
Table 1 Xylan mass balance throughout pretreatment and wash

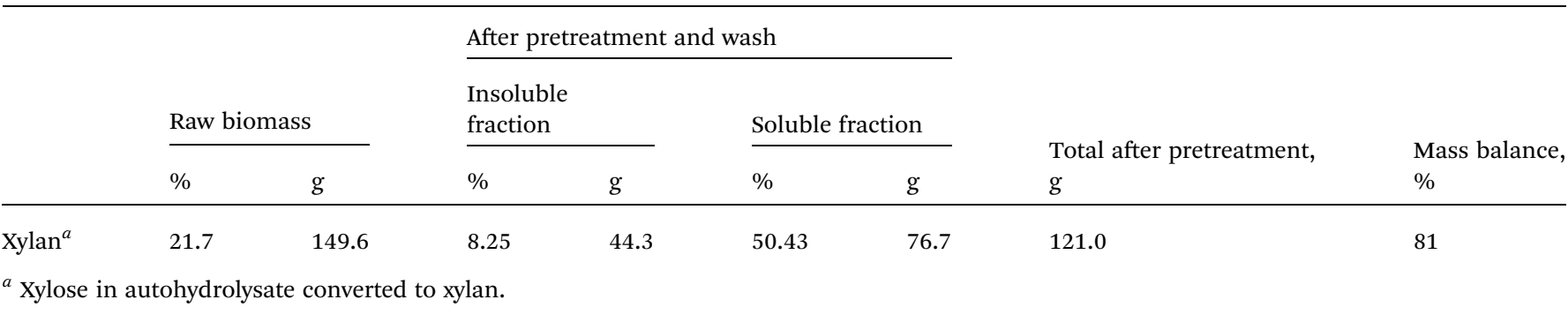

from "total" xylose). "Free" and "tied" XOS can be calculated. "Tied" XOS was calculated by subtracting "free" XOS (detected by IC with no acid hydrolysis) from "total" XOS (determined by difference in xylose concentration before and after acid hydrolysis). When calculated in this way, the "tied" XOS fraction represents the fraction of XOS that is likely to be associated with lignin via LCC. Note that the "tied" XOS fraction could include xylose units attached to lignin if such structures are present.

The concentrations of "free" and "total" xylose were $\sim 5 \mathrm{mg}$ $\mathrm{ml}^{-1}$ and $\sim 21 \mathrm{mg} \mathrm{ml}^{-1}$ in the autohydrolysate liquor, respectively $(21.7 \%$ and $100 \%$ of total xylose, respectively). The concentration of "total" XOS was calculated to be $\sim 16 \mathrm{mg} \mathrm{ml}^{-1}$ (78.3\% of total xylose; Table 3 ).

"Free XOS" includes xylooligosaccharides with a DP of 2 and above and was present at a concentration of $11.1 \mathrm{mg} \mathrm{ml}^{-1}$ ( $\sim 69 \%$ of total XOS). The concentration of "tied XOS" was $4.91 \mathrm{mg} \mathrm{ml}^{-1}(\sim 31 \%$ of total XOS). These results indicate that almost one-third (by mass) of the XOS present in the Miscanthus autohydrolysate was present as "tied XOS".

2D-HSQC NMR analysis of hydrophobic resin adsorbate produced from Miscanthus autohydrolysate showed the presence of correlations corresponding to both lignin and carbohydrates, as expected. Additionally, correlations associated with $\gamma$-uronosyl ester and phenyl glycoside LCC structures were observed, in accordance with previous reports (Fig. 1). ${ }^{21,32}$ The demonstrated presence of LCC linkages provides support to the claim that the "tied" XOS fraction is associated with lignin. A signal associated with the benzyl ether type LCC linkage was not observed. This is believed to be due to the cleavage of ether linkages in the mild acid conditions of autohydrolysis.

In addition to LCC, clear signals associated with xylan and notably, 4-O-methyl glucuronic acid side-groups, were observed (GluA, shown in purple). The presence of these signals indicates that there are branched xylans present in the adsorbate. These branched xylans may be expected to be included in the "tied" XOS fraction as well.

Based on the lignin and "tied" XOS content in the autohydrolysate, the number of "tied" xylose units per lignin C9 unit was estimated to be between two and three. Previous reports ${ }^{21}$ have estimated that LCC linkages account for $~ 9.5$ C9 units per 100 C9 units in lignin recovered from autohydrolysis liquor of non-wood biomass (sugarcane bagasse). An earlier study estimated that the total LCC linkages are in the range of at least five to more than fifteen units per 100 C9 units, depending on the biomass species and LCC isolation method. ${ }^{32}$ These values together imply that the average DP of XOS attached to LCC is quite high ( $>10$ units). Based on these results, it is apparent that techniques for the selective fractionation of lignin from autohydrolysate must be investigated with consideration for the significant amount of XOS that is associated with lignin.

\subsection{Adsorption of autohydrolysate on hydrophobic resin}

Hydrophobic resin was investigated for the selective separation of non-carbohydrate components from XOS in Miscanthus autohydrolysate with particular focus on the fate of XOS (both "free" and "tied"). Almost $\sim 90 \%$ of the dissolved lignin was removed from the autohydrolysate after 30 minutes of contact time (Fig. 2) in agreement with previous reports. ${ }^{21} \mathrm{~A}$ bit less than $20 \%$ of the free xylose was removed during this process, however, a large amount of "free" XOS was removed by the hydrophobic resin treatment. Interestingly, all of the "tied" XOS was removed, providing further evidence that the "tied" XOS fraction may be associated with lignin. This result further implies that cleavage of LCC bonds could be advantageous for improved recovery of XOS from autohydrolysates.

Table 2 Characterization of autohydrolysate including oligosaccharides and organic acids (\% of solids in AH). Standard deviations of replicates were below 0.07 for all tests conducted and are therefore not included

\begin{tabular}{|c|c|c|c|c|c|c|c|c|c|c|c|c|c|c|}
\hline & \multicolumn{4}{|c|}{ Monomers } & \multicolumn{4}{|c|}{ Oligosaccharides } & FA & AA & $\mathrm{HMF}$ & Furfural & Lignin & Unknown \\
\hline$\%$ & $1.1 \%$ & $14.9 \%$ & $0.5 \%$ & $6.5 \%$ & $4.8 \%$ & $37.3 \%$ & $1.5 \%$ & $2.4 \%$ & $2.6 \%$ & $15.3 \%$ & $0.05 \%$ & $0.1 \%$ & $7.8 \%$ & $5.1 \%$ \\
\hline
\end{tabular}

${ }^{a}$ Quantity of unknown solids was calculated by difference from total dissolved solids present in 100 ml of autohydrolysate (determined by oven drying). 
Table 3 Characterization of autohydrolysate xylooligosaccharides by ion chromatography. "Tied XOS" are believed to be associated with LCC

\begin{tabular}{llc}
\hline & & $\begin{array}{l}\% \text { (of } \\
\text { OD solid) }\end{array}$ \\
\hline Free xylose & 5.12 & $21.7 \%$ \\
Free X2 & 2.29 & $9.7 \%$ \\
Free X3 & 1.73 & $7.3 \%$ \\
Free X4 & 1.51 & $6.4 \%$ \\
Free X5 & 1.48 & $6.3 \%$ \\
Free X6+ & 4.09 & $17.3 \%$ \\
Tied XOS & 4.91 & $20.8 \%$ \\
Lignin & 1.94 & $8.2 \%$ \\
& & \\
\hline
\end{tabular}

In conducting an overall mass balance of XOS and lignin throughout the hydrophobic resin treatment process, $100 \%$ of the "free" xylose and xylobiose (X2) in the raw autohydrolysate was accounted for in the "clean" autohydrolysate and the resin adsorbate recovered with methanol. However, the total mass balance for the higher DP XOS and for lignin was not as good. Only $\sim 60 \%$ of starting X6+ XOS was accounted for in the "clean" autohydrolysate and adsorbate streams after treatment. Similarly, the overall mass balance for lignin was $\sim 70 \%$. This result implies that some high DP XOS and lignin remains adsorbed to the hydrophobic resin even after extensive washing with water and then methanol.

\subsection{Effect of XOS degree of polymerization (DP) and water wash}

The removal of XOS by hydrophobic resin treatment was highly dependent on the XOS DP (Fig. 2). The amount of XOS removed increased with increasing XOS DP. To minimize the removal of xylose and XOS from the autohydrolysate liquor, an extensive water wash was employed following adsorption. The resin, containing adsorbed compounds, was washed five times with $100 \mathrm{~mL}$ of DI water each time to remove any loose or weakly adsorbed compounds. The extensive wash significantly improved recovery of xylose and XOS. However, large amounts of XOS still remained adsorbed onto the resin (Fig. 2).

After washing, $100 \%$ of the xylose was recovered, as was most of the xylobiose and xylotriose (94\% and $74 \%$ of original, respectively). The removal of XOS with DPs of four and above was more significant. Only $26 \%$ of the (starting) XOS with a DP of six and above was recovered even after extensive washing. Extensive washing was found to have little impact on the removal of lignin.

To confirm adsorption of free XOS onto hydrophobic resin, and to show that DP influences adsorption, oligosaccharide model compounds (cellobiose and dextrans) were contacted with hydrophobic resin. Since cellobiose is only a dimer, adsorption of a dextran ( $1500 M_{\mathrm{r}}$; Sigma) was tested as well. Results showed that the amount of oligosaccharide removed by adsorption with hydrophobic resin (as a percent of starting oligosaccharide) increased linearly with increasing DP (Fig. 3), confirming the results of autohydrolysate adsorption tests.

As has been demonstrated, a large portion of the XOS removed by hydrophobic resin treatment is likely associated with lignin, however, a large amount of "free" XOS is removed as well. The interaction of oligosaccharides (which are typically considered hydrophilic) with hydrophobic surfaces is supported by literature. Previous research has indicated that certain conformations of oligosaccharides show amphiphilic behavior. ${ }^{33,34}$ These structural conformations are such that all hydrogens present to one face and all hydroxyl groups present to the other. $\beta$-1-4-D-linked oligosaccharides, such as XOS, are less capable of entering these conformations compared with $\alpha-1-4-D-l i n k e d$ oligosaccharides. However, xylose is one of the more hydrophobic monosaccharides. Additionally, increased DP improves the capacity of oligosaccharides to enter amphiphilic conformations, in support of our findings. ${ }^{33,34}$

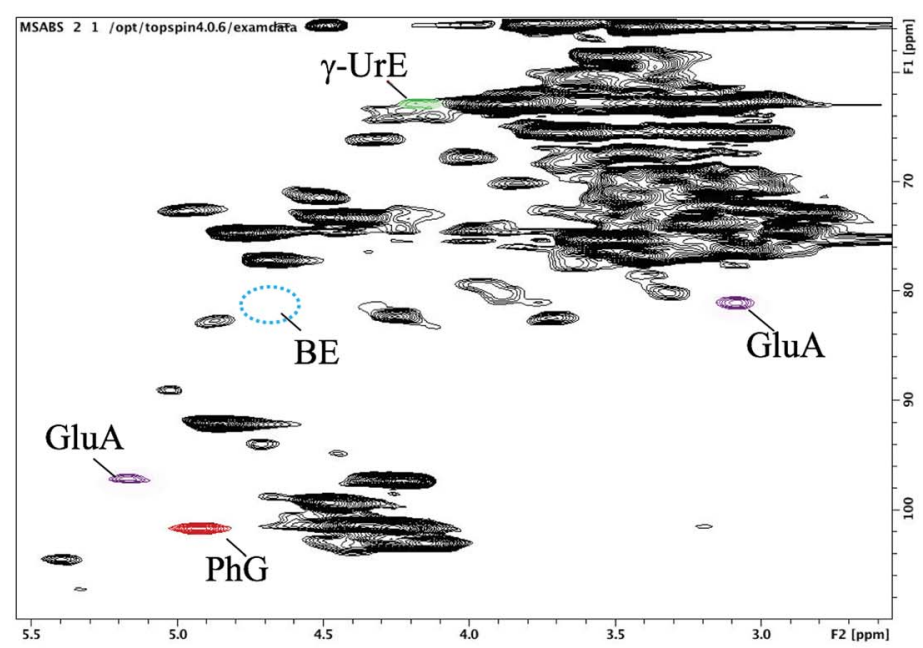

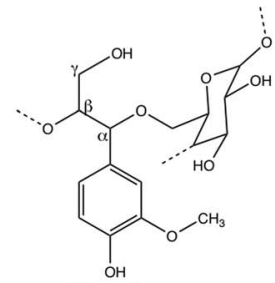

Benzyl Ether (BE)

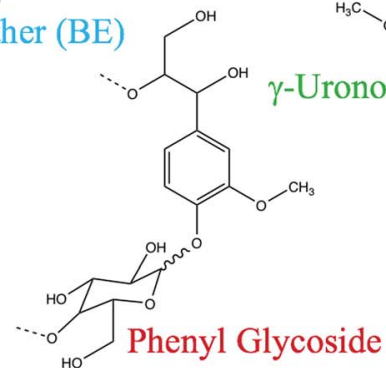

Fig. 1 2D-HSQC NMR spectrum of hydrophobic adsorbate from Miscanthus autohydrolysate showing the presence of cross-correlated peaks associated with uronosyl ester and phenyl glycosidic LCC linkages. 


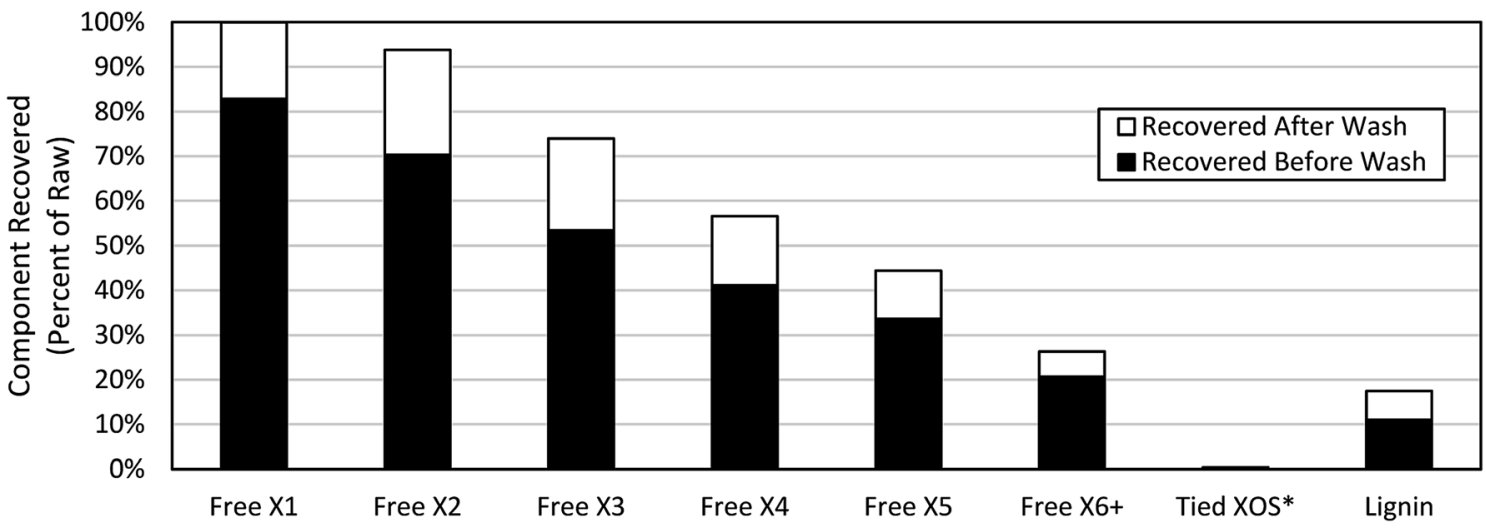

Fig. 2 Recovery of free XOS, tied XOS, and lignin during hydrophobic resin treatment. White bar represents additional component recovered during extensive washing *No wash performed.

In accordance with our findings, previous reports on the treatment of lignocellulosic autohydrolysates with hydrophobic resin also indicated removal of XOS. Narron et al. reported substantial loss of XOS during resin treatment $(\sim 30 \%$ of oligomeric xylose). ${ }^{21}$ Huang et al. employed an extensive wash to minimize the removal of XOS, but $\sim 10 \%$ of XOS were still lost. ${ }^{29}$ Narron et al. hypothesized that LCC may be to blame for XOS removal, however, our results indicated that even XOS that was definitely not associated with lignin was removed by hydrophobic resin treatment. Previous reports have not discussed the impact of DP on XOS removal during this process.

\subsection{Impact of hydrophobic resin physical properties on XOS adsorption}

Physical properties of the hydrophobic resin, such as pore-size, specific surface area, porosity, and particle size were hypothesized to impact XOS adsorption due to the potential for physical interaction between oligosaccharide chains and the resin structure. Three different hydrophobic resins were tested for removal of non-carbohydrate components and recovery of XOS from Miscanthus AH. The chemical compositions of the three hydrophobic resins were identical. However, the physical properties were all different between the three resins. Therefore, observed differences in adsorption could be attributed to a combination of resin properties.

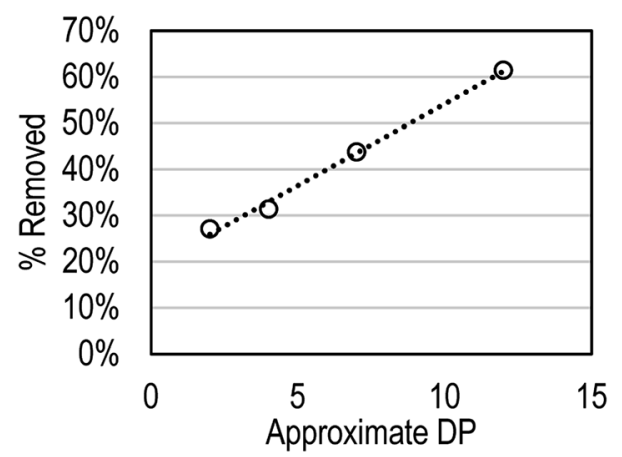

Fig. 3 Impact of DP on model oligosaccharide removal by hydrophobic resin (3 $\mathrm{mg} \mathrm{ml}^{-1}$ starting concentration).
Results indicated that the average pore size of the resin had very little impact on adsorption of XOS regardless of DP (Fig. 4). Interestingly, however, the physical properties of the hydrophobic resin were observed to impact the removal of lignin during adsorption. Adsorption of autohydrolysate by XAD 4 resulted in the removal of almost 25 percentage points less lignin (data not shown). While the use of hydrophobic resin with different physical properties was not altogether irrelevant to the adsorption process, adsorption of XOS appears to be independent of the physical structure of the hydrophobic resin within the range tested in this study (Fig. 4).

\subsection{Impact of pH on adsorption of autohydrolysate onto hydrophobic resin}

The effect of $\mathrm{pH}$ on the adsorption of lignin by hydrophobic resin was tested in order to improve the selectivity of the resin to lignin adsorption. The main mechanism by which solutes are adsorbed onto the XAD's aromatic surface are hydrophobic interactions. However, $\mathrm{pH}$ is expected to have an important impact on the charge of the dissolved polymers. Lignin and hemicelluloses are known to feature ionizable functional groups. Furthermore, pH may have an impact on oligosaccharide conformation in solution. Since lignin is known to

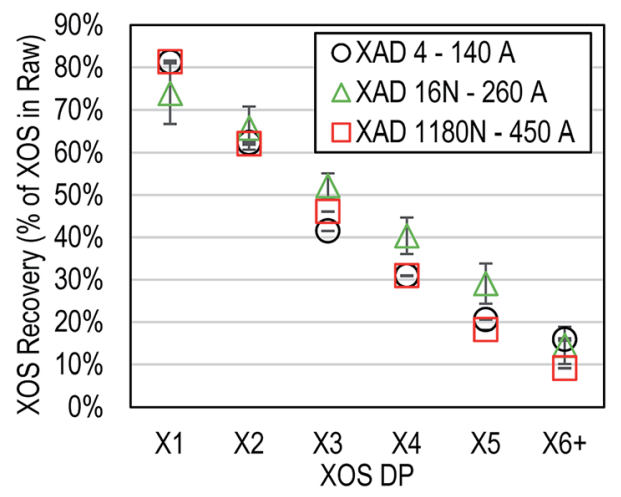

Fig. 4 Impact of XAD average pore diameter onrecovery of XOS (as a function of degree of polymerization; DP). Error bars represent one standard deviation above and below the average. 


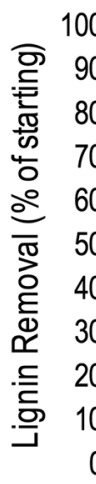

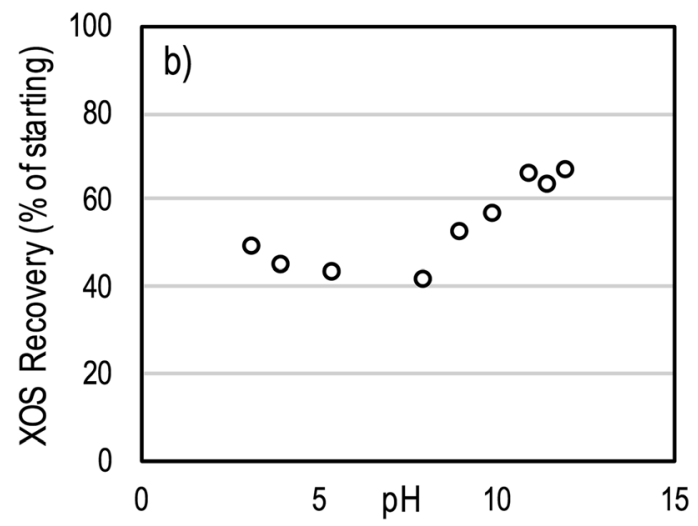

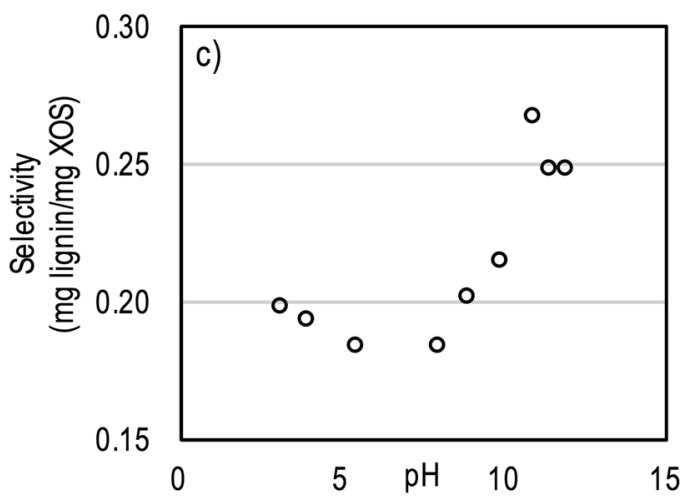

Fig. 5 Impact of $\mathrm{pH}$ on (a) lignin removal and (b) "free" XOS recovery by XAD resin. (c) Adsorption selectivity (mg lignin removed by XAD/mg XOS removed by XAD) as a function of $\mathrm{pH}$. "Free" XOS recovery is for an average of all degrees of polymerization. No "extensive" wash was used during these experiments.

precipitate at reduced $\mathrm{pH}$, the $\mathrm{pH}$ was only increased (from 3.2 up to a maximum of 12).

The $\mathrm{pH}$ of the autohydrolysate had an impact on both lignin and XOS adsorption onto hydrophobic resin (Fig. 5). Increased $\mathrm{pH}$ reduced the adsorption of lignin onto the resin from $\sim 90 \%$ at a pH of 3.2 to $\sim 70 \%$ at a pH of 12 (as a percent of lignin in starting autohydrolysate). Increased $\mathrm{pH}$ initially reduced recovery of XOS, but above a $\mathrm{pH}$ of $\sim 8$ the recovery of XOS increased dramatically. Utilizing the lignin and XOS removal results, a selectivity value was determined for each $\mathrm{pH}$ based on eqn (1).

$$
\text { Adsorption selectivity }=\frac{\text { Lignin } \operatorname{removed}(\mathrm{mg} / \mathrm{ml})}{\operatorname{XOS} \operatorname{removed}(\mathrm{mg} / \mathrm{ml})}
$$

Based on this analysis, the highest selectivity for the hydrophobic resin adsorption process occurred at high $\mathrm{pH}$ (12) due to the reduced removal (increased recovery) of XOS. However, the reduced removal of lignin at high $\mathrm{pH}$ makes this condition unfavorable.

Motivated by the results of $\mathrm{pH}$ testing, we additionally tested a three step process wherein initial adsorption was conducted at pH 3.2 followed by removal of adsorbates by methanol. Then, following the methanol wash, a high $\mathrm{pH}$ (12) water wash was conducted to remove XOS still adsorbed on the hydrophobic resin. The high $\mathrm{pH}$ water wash was found to preferentially remove higher DP XOS (X6-X12). However, most (>95\%) of the adsorbed XOS that was not removed by methanol also remained attached to the resin surface even after washing at high $\mathrm{pH}$.

\section{Conclusions}

In this study, xylooligosaccharides (XOS) in raw Miscanthus autohydrolysate were found to be present in a complex mixture including a broad range of DPs and around 30\% present as "tied" XOS, either associated with lignin or branched. Treatment with hydrophobic resin resulted in the complete removal of the "tied" portion of XOS. However, "free" XOS was found to be removed by resin treatment as well, at rates that were highly dependent on DP. The physical properties (pore-size, specific surface area, porosity, and particle size) of the resin were found to have no impact on adsorption of XOS. $\mathrm{pH}$ was found to impact both XOS and lignin adsorption to hydrophobic resin. High $\mathrm{pH}(\sim 12)$ improved the adsorption selectivity to lignin removal, but hampered the total removal of lignin. A high $\mathrm{pH}$ wash was successful at removing only a small portion of the high DP XOS that remained adsorbed after water washing of the resin.

Based on the results of these studies, it can be concluded that strongly hydrophobic resin may not be ideal for purification of higher DP XOS (>4), which has the capacity to behave hydrophobically in certain environments. The relatively lower 
removal of low DP XOS during adsorption indicates the potential for a viable process utilizing hydrophobic resin if all of the $\mathrm{XOS}$ in $\mathrm{AH}$ is reduced to a DP of $2-4$ prior to treatment with hydrophobic resin. However, further development is needed before such a process is economically viable for purification of autohydrolysate streams for prebiotic applications.

\section{Conflicts of interest}

There are no conflicts of interest to declare.

\section{Acknowledgements}

This work was supported by the USDA National Institute of Food and Agriculture under the award no. 2017-67030-26872. In addition, a portion of the work was supported by E. J. "Woody" Rice Professorship Endowment.

\section{References}

1 A. F. A. Carvalho, P. de O. Neto, D. F. da Silva and G. M. Pastore, Xylo-Oligosaccharides from Lignocellulosic Materials: Chemical Structure, Health Benefits and Production by Chemical and Enzymatic Hydrolysis, Food Res. Int., 2013, 51(1), 75-85, DOI: 10.1016/j.foodres.2012.11.021.

2 I. Jain, V. Kumar and T. Satyanarayana, Xylooligosaccharides: An Economical Prebiotic from Agroresidues and Their Health Benefits, Indian J. Exp. Biol., 2015, 53(3), 131-142.

3 A. K. Samanta, N. Jayapal, C. Jayaram, S. Roy, A. P. Kolte, S. Senani and M. Sridhar, Xylooligosaccharides as Prebiotics from Agricultural By-Products: Production and Applications, Bioact. Carbohydr. Diet. Fibre, 2015, 5(1), 6271, DOI: 10.1016/j.bcdf.2014.12.003.

4 S. Patel and A. Goyal, The Current Trends and Future Perspectives of Prebiotics Research: A Review, 3 Biotech, 2012, 2(2), 115-125, DOI: 10.1007/s13205-012-0044-x.

5 C. Hong, D. Corbett, R. Venditti, H. Jameel and S. Park, Xylooligosaccharides as Prebiotics from Biomass Autohydrolyzate, LWT-Food Sci. Technol., 2019, 111, 703710, DOI: 10.1016/j.lwt.2019.05.098.

6 P. K. Gupta, P. Agrawal, P. Hegde, N. Shankarnarayan, S. Vidyashree, S. A. Singh and S. Ahuja, Xylooligosaccharide - A Valuable Material From Waste to Taste: A Review, J. Environ. Res. Dev., 2016, 10(3), 555-563.

7 Global Xylooligosaccharides Xos Market Report 2018 - Market Reports World, https://www.marketreportsworld.com/global-xylooligosaccharides-xos-market-report-201810949853, accessed May 6, 2019.

8 T. E. Amidon and S. Liu, Water-Based Woody Biorefinery, Biotechnol. Adv., 2009, 27(5), 542-550.

9 T. E. Amidon, B. Bujanovic, S. Liu and J. R. Howard, Commercializing Biorefinery Technology: A Case for the Multi-Product Pathway to a Viable Biorefinery, Forests, 2011, 2(4), 929-947, DOI: 10.3390/f2040929.

10 P. Alvira, E. Tomás-Pejó, M. Ballesteros and M. J. Negro, Pretreatment Technologies for an Efficient Bioethanol Production Process Based on Enzymatic Hydrolysis: A
Review, Bioresour. Technol., 2010, 101(13), 4851-4861, DOI: 10.1016/j.biortech.2009.11.093.

11 N. Mosier, C. Wyman, B. Dale, R. Elander, Y. Y. Lee, M. Holtzapple and M. Ladisch, Features of Promising Technologies for Pretreatment of Lignocellulosic Biomass, Bioresour. Technol., 2005, 96(6), 673-686, DOI: 10.1016/ j.biortech.2004.06.025.

12 R. H. Narron, H. Kim, H. Chang, H. Jameel and S. Park, Biomass Pretreatments Capable of Enabling Lignin Valorization in a Biorefinery Process, Curr. Opin. Biotechnol., 2016, 38, 39-46, DOI: 10.1016/j.copbio.2015.12.018.

13 L. A. R. Batalha, Q. Han, H. Jameel, H. Chang, J. L. Colodette and F. J. Borges Gomes, Production of Fermentable Sugars from Sugarcane Bagasse by Enzymatic Hydrolysis after Autohydrolysis and Mechanical Refining, Bioresour. Technol., 2015, 180, 97-105, DOI: 10.1016/j.biortech.2014.12.060.

14 G. Garrote, H. Dominguez and J. C. Parajo, Mild Autohydrolysis: An Environmentally Friendly Technology for Xylooligosaccharide Production from Wood, J. Chem. Technol. Biotechnol., 1999, 74(11), 1101-1109.

15 A. Mittal, S. G. Chatterjee, G. M. Scott and T. E. Amidon, Modeling Xylan Solubilization during Autohydrolysis of Sugar Maple and Aspen Wood Chips: Reaction Kinetics and Mass Transfer, Chem. Eng. Sci., 2009, 64(13), 30313041, DOI: 10.1016/j.ces.2009.03.011.

16 D. B. Corbett, A. J. Murphy and B. Bujanovic, Chemical Composition and Hot Water Extraction of Apricot Seed Shells: Effect on the Combustion Energy and Lignin Content, Syracuse, New York, 2013.

17 M.-H. Chen, M. J. Bowman, B. S. Dien, K. D. Rausch, M. E. Tumbleson and V. Singh, Autohydrolysis of Miscanthus $x$ Giganteus for the Production of Xylooligosaccharides (XOS): Kinetics, Characterization and Recovery, Bioresour. Technol., 2014, 155, 359-365, DOI: 10.1016/j.biortech.2013.12.050.

18 P. Ligero, J. C. van der Kolk, A. de Vega and J. E. G. van Dam, Production of Xylo-Oligosaccharides from Miscanthus $\mathrm{x}$ Giganteus By Autohydrolysis, BioResources, 2011, 6(4), 4417-4429.

19 R. El Hage, L. Chrusciel, L. Desharnais and N. Brosse, Effect of Autohydrolysis of Miscanthus x Giganteus on Lignin Structure and Organosolv Delignification, Bioresour. Technol., 2010, 101(23), 9321-9329, DOI: 10.1016/ j.biortech.2010.06.143.

20 G. Garrote, E. Falqué, H. Domínguez and J. C. Parajó, Autohydrolysis of Agricultural Residues: Study of Reaction Byproducts, Bioresour. Technol., 2007, 98(10), 1951-1957, DOI: 10.1016/j.biortech.2006.07.049.

21 R. H. Narron, H. Chang, H. Jameel and S. Park, Soluble Lignin Recovered from Biorefinery Pretreatment Hydrolyzate Characterized by Lignin-Carbohydrate Complexes, ACS Sustainable Chem. Eng., 2017, 5(11), 10763-10771, DOI: 10.1021/acssuschemeng.7b02716.

22 P. Ye, L. Cheng, H. Ma, B. Bujanovic, M. J. Goundalkar and T. E. Amidon, Biorefinery with Water in, The Role of Green Chemistry in Biomass Processing and Conversion, ed. H. Xie and N. Gathergood, John Wiley \& Sons, Inc., New York, USA, 2012, pp. 135-180. 
$23 \mathrm{~J} . \mathrm{Li}$ and G. Gellerstedt, Improved Lignin Properties and Reactivity by Modifications in the Autohydrolysis Process of Aspen Wood, Ind. Crops Prod., 2008, 27(2), 175-181, DOI: 10.1016/j.indcrop.2007.07.022.

24 M. J. Vázquez, G. Garrote, J. L. Alonso, H. Domínguez and J. C. Parajó, Refining of Autohydrolysis Liquors for Manufacturing Xylooligosaccharides: Evaluation of Operational Strategies, Bioresour. Technol., 2005, 96(8), 889-896, DOI: 10.1016/j.biortech.2004.08.013.

$25 \mathrm{C}$. Wu, The Potential of Pre-Hydrolysis Liquor from the Dissolving Pulp Process as Recovery Source of Xylooligosaccharide - A Mini-Review, BioResources, 2016, 11(3), 7917-7927, DOI: 10.15376/biores.11.3.wu.

26 R. Vegas, S. Luque, J. R. Alvarez, J. L. Alonso, H. Domínguez and J. C. Parajó, Membrane-Assisted Processing of Xylooligosaccharide-Containing Liquors, J. Agric. Food Chem., 2006, 54(15), 5430-5436.

27 Q. P. Yuan, H. Zhang, Z. M. Qian and X. J. Yang, Pilot-Plant Production of Xylo-Oligosaccharides from Corncob by Steaming, Enzymatic Hydrolysis and Nanofiltration, J. Chem. Technol. Biotechnol., 2004, 79(10), 1073-1079, DOI: 10.1002/jctb.1071.

28 AMBERLITE XAD 16N, Dow Product Data Sheet Form No. 177-03120-0313, DOW.

29 C. Huang, B. Jeuck, J. Du, Q. Yong, H. Chang, H. Jameel and R. Phillips, Novel Process for the Coproduction of Xylo-
Oligosaccharides, Fermentable Sugars, and Lignosulfonates from Hardwood, Bioresour. Technol., 2016, 219, 600-607, DOI: 10.1016/j.biortech.2016.08.051.

30 A. Sluiter, B. Hames, R. Ruiz, C. Scarlata, J. Sluiter and D. Templeton, Determination of Sugars, Byproducts, and Degradation Products in Liquid Fraction Process Samples, NREL National Renewable Energy Laboratory, 2008.

31 B. Yang and C. E. Wyman, Characterization of the Degree of Polymerization of Xylooligomers Produced by Flowthrough Hydrolysis of Pure Xylan and Corn Stover with Water, Bioresour. Technol., 2008, 99(13), 5756-5762, DOI: 10.1016/ j.biortech.2007.10.054.

32 M. Balakshin, E. Capanema, H. Gracz, H. Chang and H. Jameel, Quantification of Lignin-Carbohydrate Linkages with High-Resolution NMR Spectroscopy, Planta, 2011, 233(6), 1097-1110.

33 C. Sivakama Sundari, B. Raman and D. Balasubramanian, Hydrophobic Surfaces in Oligosaccharides: Linear Dextrims Are Amphiphilic Chains, Biochim. Biophys. Acta, Biomembr., 1991, 1065(1), 35-41, DOI: 10.1016/00052736(91)90007-U.

34 C. S. Sundari and D. Balasubramanian, Hydrophobic Surfaces in Saccharide Chains, Prog. Biophys. Mol. Biol., 1997, 67(2), 183-216, DOI: 10.1016/S0079-6107(97)00016-3. 